\title{
Der Sommer treibt Blüten
}

\author{
ROLAND RUdolPH \& HiLKe STEINECKE
}

\begin{abstract}
Hot and sunny periods during the past few years led to an increase in flowering of certain (sub-)tropical plants cultivated at the Palmengarten. Especially spectacular were certain Agave species with large inflorescences, and some cacti that were covered with dozens of flowers. The paper describes some of the extraordinarily rich-flowering species from our living collections.
\end{abstract}

\section{Zusammenfassung}

Heiß-sonnige Witterungsperioden der letzten Jahre haben im Palmengarten zunehmend eine reiche Blüte wärmeliebender Arten gefördert. Auffällig waren Agaven mit ihren großen Blütenständen oder Kakteen, die mit Dutzenden von Blüten übersäht waren. Einige von ihnen werden vorgestellt.

\section{Einleitung}

In den letzten Jahren gab es im Frühjahr bzw. Sommer auffällig sonnig-heiße Witterungsperioden mit Temperaturen über $30^{\circ} \mathrm{C}$. Manch eine Pflanze bekommt dann Stress, vor allem, wenn der Boden austrocknet und nicht gegossen wird. Anderseits profitieren sonnen- und wärmehungrige Arten von der Klimaerwärmung, darunter zahlreiche Wüstenpflanzen. Dies äußerte sich im Sommerhalbjahr 2008 in einer besonders üppigen Blüte verschiedener Arten sowohl in den Gewächshäusern als auch draußen im Sommer-Sukkulentengarten.

\section{Freie Fahrt für Agaven-Blüitenstände}

Die Gattung Agave (inkl. Manfreda, Polianthes) umfasst ca. 220 Arten. Sie sind in Mittel- und Südamerika heimisch, viele Arten findet man in Mexiko. Zu den bekanntesten Vertretern gehören sicherlich die Amerikanische Agave ( $A$. americana), die im Mittelmeergebiet an vielen Stellen verwildert vorkommt, die Faser liefernde Sisal-Agave (A. sisalana) sowie A. tequilina, aus deren Saft das alkoholische Getränk Tequila hergestellt wird. Agaven faszinieren aufgrund ihres Lebensrhythmus, denn die Blattrosette einiger Arten wird oft mehrere Jahrzehnte alt, bis endlich ein riesiger, bis $10 \mathrm{~m}$ hoher Blütenstand austreibt. Nach der Fruchtreife sterben die Mutterpflanzen ab, ähnlich wie die Bananenstauden. Gelegentlich werden im Bereich des Blütenstandes auch Kindel zur vegetativen Vermehrung gebildet. Häufiger kommt eine reichliche Kindelbildung am Grunde der Rosetten vor.
Der Palmengarten besitzt eine der größten Agavensammlungen in Europa. Rund 80 Arten, also knapp 1/3 aller bekannten Agaven-Spezies, sind meist durch mehrere Individuen vertreten. Sie gedeihen im Tropicarium (vor allem im Halbwüsten-Haus) und in der botanischen Sammlung. Im Sommerhalbjahr werden einige von ihnen in den wie eine hügelige Landschaft gestalteten Bereich des Sommer-Sukkulentengartens integriert und ca. 100 Kübel, die zur dokumentierten Agavensammlung gehören, um den Sukkulentengarten herum ausgestellt. Einige Exemplare sind bereits mehrere Jahrzehnte in Obhut des Palmengartens, von vielen gibt es eigene Nachzuchten. Teilweise sind die Pflanzen so groß und schwer, dass die Kübel nur mit einem Gabelstapler transportiert werden können. Herausragend sind zwei übermannshohe Exemplare von Agave americana, die über 50 Jahre alt sind. Beeindruckend ist auch die Vielfalt der Blattformen, die von schmal, glattrandig bis breit und stechend bestachelt reicht. Die leicht eingerollten, am Rand mit spitz dreieckigen Stacheln besetzten grünen Blätter von $A$. shawii subsp. goldmaniana erinnern an Krokodilschnauzen.

Jedes Jahr kommen regelmäßig einzelne Agaven zur Blüte. Stehen die Pflanzen im Freiland oder im relativ hohen Halbwüsten-Haus, ist das kein Problem, denn dem Wachstum sind dort keine Grenzen gesetzt. Sehr gut konnte beispielsweise von der Brücke im Halbwüsten-Haus beobachtet werden, wie sich während der Sommermonate allmählich der verzweigte Blütenstand einer Agave parasana streckte. Die Blüten dieser 

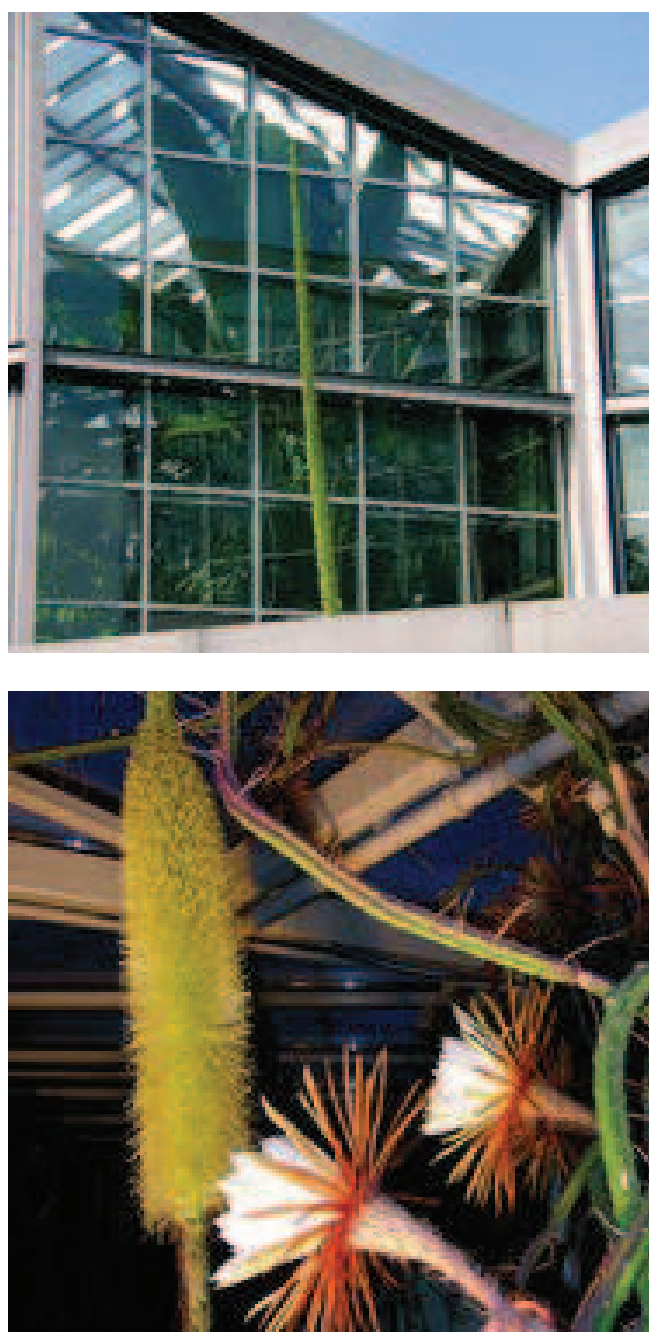

Art sind hellgelb gefärbt. Sehr gut konnte daneben die Infloreszenz von $A$. striata verglichen werden. Sie stammt aus dem Nordosten Mexikos und bildet eine dichte Rosette aus langen, sehr schmalen und stacheligen Blättern. Ihr Blütenstand ist vergleichsweise schmal und unverzweigt. Die Blüten sind grünlich-gelb. Auffällig sind die langen Filamente und die rotbraunen Staubbeutel, die aus der Blüte heraushängen. Zudem zeigten sich im vorigen Sommer im selben Haus zwei Blütenstände von $A$. victoria-reginae. Diese Agave mit blaugrauen Blättern und schwarzen Stacheln ist sehr attraktiv.

Problematischer ist die Entwicklung von Blütenständen in den niedrigen Häusern der bota- nischen Sammlung. Ihr Wachstum wird gehemmt, wenn sie an das Glasdach stoßen, sich verkrümmen und sich nicht bis zu ihrer maximalen Länge entfalten können. Damit sich der Blütenstand einer Agave victoria-reginae var. fernandis in der botanischen Sammlung aber doch $5 \mathrm{~m}$ hoch entfalten konnte, wurde eine Scheibe aus dem Glasdach ausgebaut. Der schlanke Blütenstand ragte nach einigen Wochen wie eine Kerze ca. $3 \mathrm{~m}$ aus dem Dach heraus. Agave victoria-reginae var. fernandis ist ein Endemit von NordostMexiko. Aufgrund ihrer attraktiven, gedrungenen Blattrosetten wurden ihre Bestände am Naturstandort geplündert. Heute wird die streng geschützte Art in vielen Gärtnereien kultiviert und ist eine häufige Zierpflanze. In direkter Nachbarschaft blühte zeitgleich auch eine Agave bracteosa. Sie kommt im Norden der Sierra Madre Oriental in Höhenlagen von 900 bis 1700 m auf Kalkfelsen und an felsigen Hängen vor. An dem etwa $2 \mathrm{~m}$ hohen und während der Blühphase über armdicken Blütenstand befanden sich Tausende cremeweißer Blüten mit etwa $6 \mathrm{~cm}$ langen Staubfäden. Sie öffneten sich mit Einbruch der Dämmerung. In der Heimat werden sie von Fledermäusen bestäubt. Pro Tag verblühten ca. $30 \mathrm{~cm}$ des Blütenstandes unseres Exemplars, so dass das eigentliche Blühereignis schnell vorbei war. Schon bei leichter Berührung rieselte massenhaft Blütenstaub aus den Pollensäcken. Durch Schütteln des Blütenstandes wird eine Selbstbestäubung gefördert, damit sich später reichlich Samen für die Nachzucht der absterbenden Mutterpflanze entwickeln.

Einen ähnlichen Habitus wie Agaven zeigen die verwandten Yucca-Arten (Palmlilien). Einige von ihnen, wie z. B. Y. filamentosa und Y. glauca, sind bei uns winterhart und im Sukkulentengarten ausgepflanzt. Ihre Blätter sind fest und faserig. Im Gegensatz zu den Agaven blühen sie jedes Jahr und sterben danach nicht ab. Im Sommer

Abb. 1 (oben): Ein Agavenblütenstand hat das Dach der botanischen Sammlung durchwachsen.

Abb. 2 (Mitte): Nächtliche Blüten von Agave bracteosa und Selenicereus pteranthus. 
2008 erfreuten sie durch eine besonders üppige Blütenpracht. In den rispig verzweigten, $1-2 \mathrm{~m}$ hohen Blütenständen befinden sich zahlreiche weiße Blüten, die etwas an große MaiglöckchenBlüten erinnern. Früchte mit Samen setzen die Pflanzen nicht an, denn bei uns gibt es keine Yuccamotten, die spezifischen Bestäuber der Palmlilien.

\section{Kakteen im Freiland und unter Glas}

Wie die Agaven profitieren einige Kakteen vom sonnigen Sommerwetter. Viele von ihnen werden von Fledermäusen bestäubt, öffnen entsprechend ihre Blüten in der Nacht und sind mitunter noch am nächsten Vormittag recht ansehnlich, insbesondere bei trübem Wetter. Ein großer Kübel mit einem Kugelkaktus (Echinopsis-Hybride) bereitete mit mehreren Blühschüben bis in den Herbst besondere Freude. Im Juli 2008 war er mit über 100 Blüten übersäht, die sich alle innerhalb von zwei Nächten öffneten. Obwohl Bienen nicht zur Bestäubung dieses Kaktus beitragen, waren die Pollenmassen für sie als Nahrungsquelle offensichtlich attraktiv. In den frühen Morgenstunden tummelten sich für ca. 15 Minuten unzählige Bienen in den Kaktusblüten, bis alle ganz plötzlich, mit dicken „Pollenhöschen“ beladen, wieder davonflogen.

In den Gewächshäusern werden von Jahr zu Jahr die Prinzessin und Königin der Nacht (Selenicereus pteranthus und S. grandiflorus) immer blühwilliger. Zugute kommt ihnen sicherlich auch, dass in den letzten Jahren das alte Glas des Daches durch neues, lichtdurchlässigeres ausgetauscht wurde. Diese Kletterkakteen sind durch die Scheibe des Gewächshauses der botanischen Sammlung von außen zu sehen, so dass man in warmen Sommernächten durchaus die Gelegenheit hat, geöffnete Blüten zu sehen. In manchen milden Sommernächten waren 40 Blüten gleichzeitig geöffnet - ein kleines nächtliches Palmen-

Abb. 3 (oben): Üppig blühende Yuccas im Sommer-Sukkulentengarten.

Abb. 4 (Mitte): In der Dämmerung öffnen sich die Blüten dieser Echinopsis-Hybride.
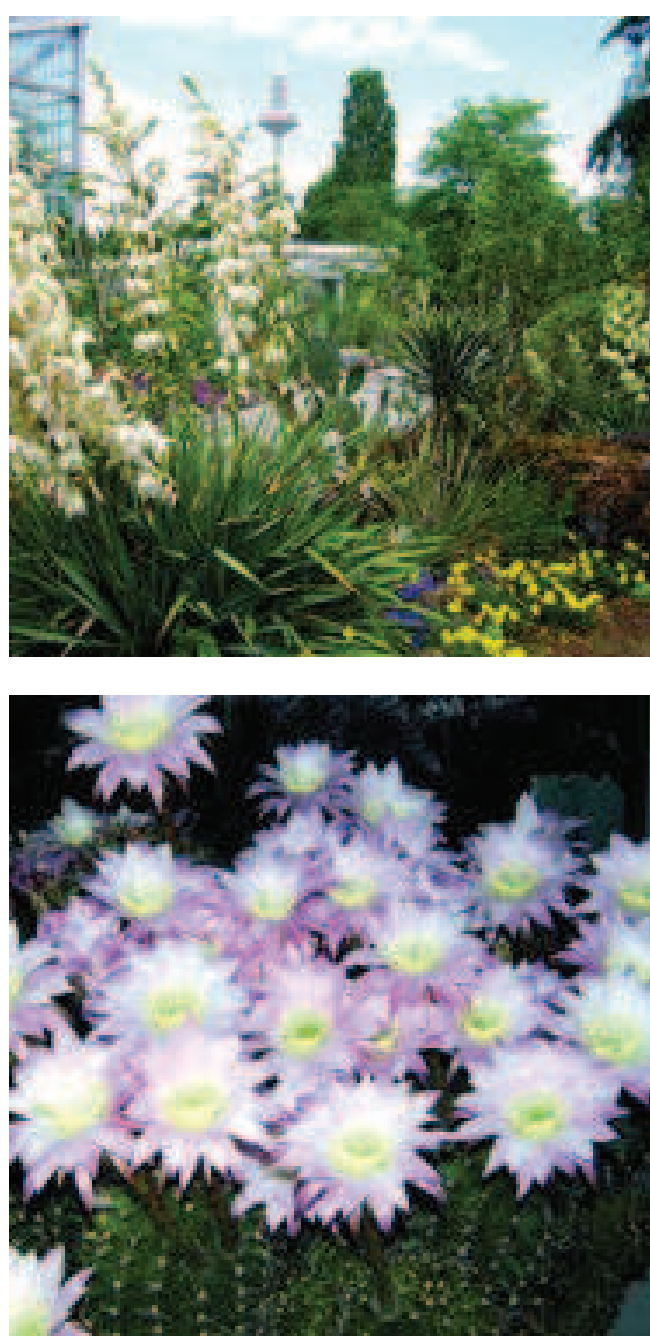

garten-Naturwunder. Da keine bestäubenden Fledermäuse zur Verfügung stehen, wurde der Pollen mit Hilfe eines Pinsels übertragen, so dass sich im Laufe der nächsten Wochen purpurviolette, etwa tomatengroße Früchte entwickeln konnten.

\section{Palmen}

Palmen sind der Inbegriff tropischer Vegetation. Bis auf wenige Arten, die bei uns im Freien überwintern können (z.B. Trachycarpus fortunei), sind die meisten wärmeliebend und meist auch sonnenhungrig. Auch wenn die Gewächshäuser relativ gleichmäßig klimatisiert sind, heizen sie sich an Sommertagen stark auf und es werden 

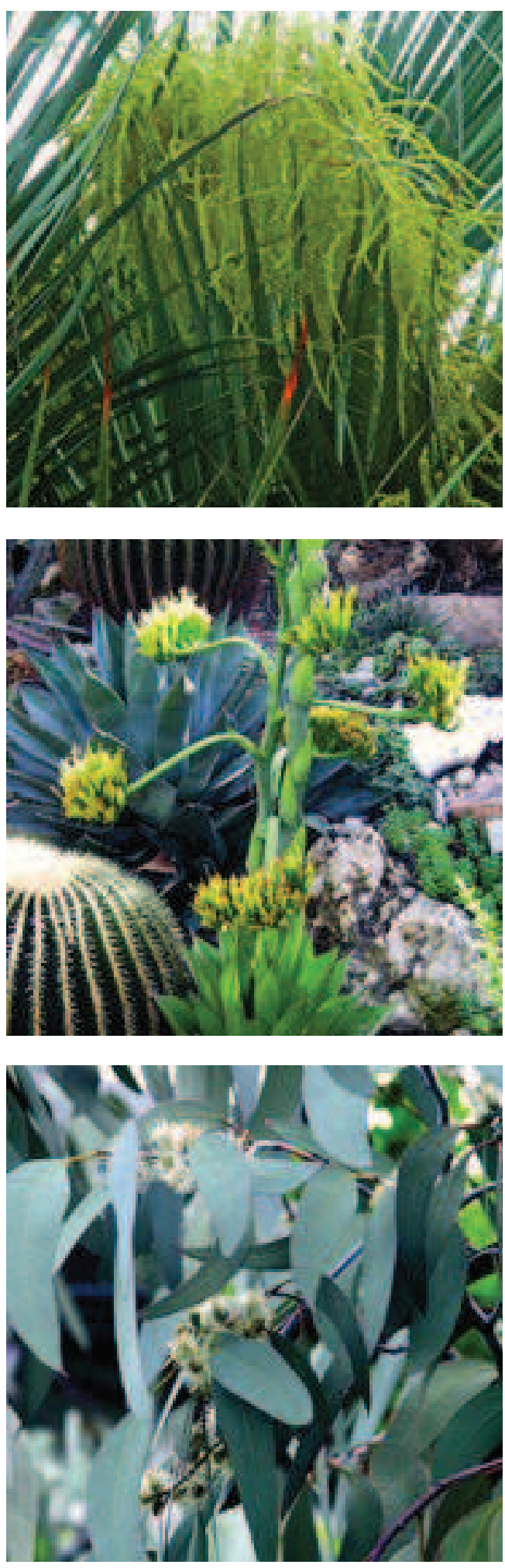

durchaus Temperaturen von $30-40^{\circ} \mathrm{C}$ erreicht. Eine Palme besonders trocken heißer Standorte ist Brahea armata (Blaue Hesperidenpalme). Sie ist in Kalifornien, Arizona und Mexiko heimisch und im Palmengarten im Halbwüsten-Haus anzutreffen. Sie fällt durch ihre festen, blaugrau bereiften großen Fächerblätter auf. Unser Exemplar ist ca. $5 \mathrm{~m}$ hoch und über 40 Jahre alt. Ältere Exemplare erreichen Höhen von $15 \mathrm{~m}$. Die sehr langen gelben Blütenstandsschweife hängen oft bis zum Boden herab. Im Sommer 2008 entwickelte das Palmengarten-Exemplar erstmals mehrere große und stark verzweigte Blütenstände und bis zum Herbst sogar Früchte. Aber auch die im Freiland ausgepflanzten Palmen bilden regelmäßig Blütenstände, darunter neben der üblicherweise als Freiland-Art kultivierten Trachycarpus fortunei auch Rhapidophyllum hystrix, Sabal minor und Serenoa repens. Die Hanfpalmen bilden reichlich Früchte, die bei Amseln als Futter sehr beliebt sind. Vögel und vermutlich auch Eichhörnchen verbreiten die Samen im Garten. An verschiedenen Stellen im Palmengarten sind Jungpflanzen von Hanfpalmen zu finden. Sie überstehen im Allgemeinen den Winter problemlos, und einige Individuen wachsen seit über 10 Jahren ohne Winterschutz. Im extrem kalten Winter 2008/2009 allerdings erlitten die Blätter starke Frostschäden.

Abb. 5 (oben): Blütenstand von Brahea armata.

Abb. 6 (Mitte): Blühende Agaven und SchwiegermuttersitzKakteen bereichern die Halbwüste im Tropicarium

Abb. 7 (unten): Rückschläge sind möglich; dieser Eukalyptus blühte erstmals im Sommer 2008, ist aber im kalten Winter 2008/2009 erfroren. Am abgesägten Stamm treibt er aber langsam wieder aus. 